\title{
AN EXPANDED METHOD TO ROBUSTLY STABILIZE UNCERTAIN NONLINEAR SYSTEMS*
}

\author{
JASON POLENDO $^{\dagger}$ AND CHUNJIANG QIAN $^{\dagger}$
}

\begin{abstract}
The current literature on the global state feedback stabilization of nonlinear systems modeled by a perturbed chain of nonlinear integrators, particularly those whose linearization about the origin may contain uncontrollable modes, essentially contains two methods: a smooth controller scheme (only under strict assumptions) and a non-smooth one. The most general of these systems could previously only be globally asymptotically stabilized by continuous time-invariant state feedback controller, where this paper shows that now at least $C^{1}$ stabilization can be achieved, upon existence, in this more general setting. This new method can be seen as not only a natural unification of the smooth and nonsmooth methods, but also a generalization to construct smoother stabilizers.
\end{abstract}

1. Introduction and Background. In this treatment we will consider the global stabilization of nonlinear power integrator systems in the form

$$
\begin{gathered}
\dot{x}_{1}=x_{2}^{p_{1}}+\phi_{1}(t, x, u) \\
\dot{x}_{2}=x_{3}^{p_{2}}+\phi_{2}(t, x, u) \\
\vdots \\
\dot{x}_{n}=u+\phi_{n}(t, x, u),
\end{gathered}
$$

where $x=\left(x_{1}, \cdots, x_{n}\right)^{T} \in \mathbb{R}^{\mathrm{n}}$ and $u \in \mathbb{R}$ are the system state and input, respectively. For $i=1, \cdots, n, \phi_{i}(t, x, u)$ is an unknown $C^{0}$ nonlinear function of the states and the control input and $p_{i} \in \mathbb{R}_{\text {odd }}^{+}:=\{q \in \mathbb{R}: q>0$ and $q$ is a ratio of odd integers $\}$, with $p_{n}$ obviously equal to one (which is not a limitation since we can easily set $v:=u^{p_{n}}$ in the case of non-unity $p_{n}$ ). The importance for studying such systems is exemplified in the papers $[16,15]$, where non-smooth state feedback controllers were used to stabilize the following underactuated, weakly coupled, unstable mechanical system introduced in $[16]$.

$$
\begin{aligned}
\dot{x}_{1} & =x_{2} \\
\dot{x}_{2} & =x_{3}^{3}+\frac{g}{l} \sin x_{1} \\
\dot{x}_{3} & =x_{4} \\
\dot{x}_{4} & =u .
\end{aligned}
$$

In recent years, the power integrator systems of the form (1) have been studied fairly extensively with various restrictions on the integrator powers and the additive

\footnotetext{
${ }^{*}$ Chunjiang Qian's research was supported in part by the U.S. National Science Foundation under grant ECCS-0239105. Jason Polendo's research was supported in part by the University of Texas at San Antonio Doctoral Fellowship and the NASA Harriett G. Jenkins Pre-Doctoral Fellowship.

$\dagger$ Dept. of Electrical and Computer Engineering, University of Texas at San Antonio, San Antonio, Texas 78249. E-mail: chunjiang.qian@utsa.edu
} 
$\phi_{i}(\cdot)$ 's, which directly influence the previous availability of smooth or non-smooth stabilizers by a constructive design scheme. And although smoothness is the more desirable result, the solutions with less restrictive assumptions on the nonlinear system are generally the ones which have achieved a non-smooth solution. In particular, a smooth stabilizer was achieved in the work [14] by the development of an 'adding a power integrator technique' under the conditions that the powers, $p_{i}$, are of a decreasing order, such that $p_{1} \geq p_{2} \geq \cdots \geq p_{n} \geq 1$ and each $\phi_{i}(\cdot)$ satisfies an appropriate lower-triangular (i.e. strict-feedback) growth condition. The power order restriction and growth condition were lifted (with $p_{i} \geq 1$ odd integers) in [15], though the tradeoff for such flexibility was non-smooth feedback stabilization. The technique from [15] was recently extended in [4] to allow for fractional odd powers less than one, with a non-smooth controller still utilized. A subset of these systems was also stabilized by a $C^{0}$ feedback control law in [18] for a linear $\phi_{i}(\cdot)$, which applied the global extension [17] of the sufficient condition for the existence of $C^{0}$ locally asymptotic stabilizer [5]. Using a homogeneous approximation of the system considered in [5], [3] constructed such locally asymptotically feedback laws.

One interesting observation is that even for the same system

$$
\dot{x}_{1}=x_{2}^{3}, \quad \dot{x}_{2}=u,
$$

the current smooth [14] and nonsmooth [15] methods yield two different stabilizers, namely

$$
u_{\text {smooth }}=-x_{1}-x_{2} \quad \text { and } \quad u_{\text {nonsmooth }}=-\left(x_{2}^{3}+x_{1}\right)^{1 / 3} .
$$

This distinction can be attributed to the different design processes of those two methods. Herein we develop a technique, in the constructive vein of the previously mentioned approaches, to unify the existing smooth and nonsmooth methods under one generalized framework. The stabilizer obtained using this new method can lead to either $u_{\text {smooth }}$ or $u_{\text {nonsmooth }}$ for system (3) by simply adjusting its parameters. Nevertheless, our methodology makes its largest contribution by allowing for at least $C^{1}$ stabilization when such freedoms exist. For instance, consider the following system

$$
\dot{x}_{1}=x_{2}^{3}+x_{1}^{2}, \quad \dot{x}_{2}=u .
$$

For $(4)$, the methods of $[15,4]$ still only produce a $C^{0}$ controller (while the smooth result of [14] is inapplicable since $x_{1}^{2}$ is not of the same order as $x_{2}^{3}$ ). However, the methodology described in this paper will enable us to offer $C^{1}$ stabilization by static state feedback for (4) and other similar systems previously stabilized only by nonsmooth or unbounded time-varying control laws.

This paper is organized in the following manner: Section 2 details our design scheme by stabilizing the system (1) in a general sense; then Section 3 describes the 
way this methodology encompasses the existing literature as special cases; and its ability to offer smoother stabilizers than previous methods is illustrated by example in Section 4.

2. A Generalized Method for Stabilizing System (1). In this section, we propose a generalized method for feedback stabilization of (1) under the following assumption:

Assumption 2.1. For $i=1, \cdots, n$, there exist constants $\tau_{1} \geq \tau_{2} \geq \cdots \geq \tau_{n}$ such that,

$$
\left|\phi_{i}(t, x, u)\right| \leq b_{i}\left(x_{1}, \ldots, x_{i}\right)\left(\left|x_{1}\right|^{\frac{r_{i}+\tau_{i}}{r_{1}}}+\left|x_{2}\right|^{\frac{r_{i}+\tau_{i}}{r_{2}}}+\cdots+\left|x_{i}\right|^{\frac{r_{i}+\tau_{i}}{r_{i}}}\right),
$$

for a smooth function $b_{i}(\cdot) \geq 0$ and $r_{i}$ defined as

$$
r_{1}=1, \quad r_{i+1}=\frac{\tau_{i}+r_{i}}{p_{i}}>0
$$

For simplicity, we assume $\tau_{i}=\frac{q_{i}}{d_{i}}$, with $q_{i}$ an even integer and $d_{i}$ an odd integer. Under this assumption, and taking into account the odd, not necessarily equivalent, powers of (1), we know the coordinate weights, $r_{i} \in \mathbb{R}_{\text {odd }}^{+}$. Note that an equivalent result will be achieved for the case when $r_{i} \notin \mathbb{R}_{\text {odd }}^{+}$.

REMARK 2.1. A distinctive feature of Assumption 2.1 is the flexibility in choosing the parameters $\tau_{i}$ 's which now can be any constants satisfying $\tau_{1} \geq \tau_{2} \geq \cdots \geq \tau_{n}$. With this newfound flexibility Assumption 2.1 becomes more general than, and actually encompasses as its special cases, the distinct assumptions made in existing results [15, 14, 10]. Specifically, when $\tau_{i}=0,1 \leq i \leq n$, condition (5) reduces to the following condition used in [15]

$$
\left|\phi_{i}(t, x, u)\right| \leq b_{i}\left(x_{1}, \ldots, x_{i}\right)\left(\left|x_{1}\right|^{\frac{1}{p_{1} \cdots p_{i-1}}}+\left|x_{2}\right|^{\frac{1}{p_{2} \cdots p_{i-1}}}+\cdots+\left|x_{i-1}\right|^{\frac{1}{p_{i-1}}}+\left|x_{i}\right|\right) .
$$

In [14] the stabilization problem was considered for system (1) where the integrator powers satisfy the structural requirements that $p_{1} \geq p_{2} \geq \cdots \geq p_{n} \geq 1$. A smooth state feedback controller was then constructed under the condition

$$
\left|\phi_{i}(t, x, u)\right| \leq b_{i}\left(x_{1}, \ldots, x_{i}\right)\left(\left|x_{1}\right|^{p_{i}}+\left|x_{2}\right|^{p_{i}}+\cdots+\left|x_{i}\right|^{p_{i}}\right),
$$

which is exactly a special case of (5) with $\tau_{i}=p_{i}-1$. Moreover, when we choose a negative $\tau$ such that $\tau_{i}=\tau<0,1 \leq i \leq n$, Assumption 2.1 can be seen to reduce to the condition used in [10] where system (1) was stabilized in a finite time. Section 3 discusses these generalities in more detail.

The following result constitutes the most general case under consideration in this paper, with the subsequent situations acting as its special cases and thereby utilizing corollaries based on our main theorem. The distinguishing factors for each scenario are the restrictions on the power integrator values, $p_{i}$, and the values $\tau_{i}$ introduced 
in Assumption 2.1. Obviously, there are many different combinations of restrictions that we could investigate, but only the most relevant ones will be discussed here, with others left as simple exercises for the interested reader. An interesting relation among the scenarios is that they yield quite different controllers in that one is non-smooth, another is guaranteed smooth, while the last is a finite-time stabilizer, however, they are still connected as being special cases of the following theorem.

Theorem 2.1. By Assumption 2.1 there exists a state feedback controller such that the nonlinear system (1) is guaranteed globally asymptotically stable ${ }^{1}$.

Proof. The inductive proof relies on the simultaneous construction of a $C^{1}$ Lyapunov function which is positive definite and proper, as well as a homogeneous-like stabilizer at each iteration.

Initial Step. Let $\sigma, \rho \in \mathbb{R}_{\text {odd }}^{+}$satisfy

$$
\sigma \geq \max _{1 \leq i \leq n}\left\{r_{i}\right\} \quad \text { and } \quad \rho \geq \max _{1 \leq i \leq n}\left\{\tau_{i}+r_{i}, \sigma\right\},
$$

where $\tau_{i}$ and $r_{i}$ are defined as in Assumption 2.1. Choose

$$
V_{1}\left(x_{1}\right)=\int_{0}^{x_{1}}\left(s^{\frac{\sigma}{r_{1}}}-0\right)^{\frac{2 \rho-\tau_{1}-r_{1}}{\sigma}} d s
$$

The time derivative of $V_{1}$ along the trajectory of (1) is

$$
\dot{V}_{1}=\frac{\partial V_{1}}{\partial x_{1}} \dot{x}_{1}=x_{1}^{\frac{2 \rho-\tau_{1}-r_{1}}{r_{1}}}\left[x_{2}^{p_{1}}+\phi_{1}(t, x, u)\right] .
$$

By Assumption 2.1,

$$
\dot{V}_{1} \leq x_{1}^{\frac{2 \rho-\tau_{1}-r_{1}}{r_{1}}} x_{2}^{* p_{1}}+x_{1}^{2 \rho / r_{1}} b_{1}\left(x_{1}\right)+x_{1}^{\frac{2 \rho-\tau_{1}-r_{1}}{r_{1}}}\left[x_{2}^{p_{1}}-x_{2}^{* p_{1}}\right] .
$$

Then, the virtual controller $x_{2}^{* p_{1}}$ defined by

$$
x_{2}^{* p_{1}}=-x_{1}^{\left(\tau_{1}+r_{1}\right) / r_{1}}\left(n+b_{1}\left(x_{1}\right)\right)=-x_{1}^{r_{2} p_{1} / r_{1}}\left(n+b_{1}\left(x_{1}\right)\right):=-x_{1}^{r_{2} p_{1} / r_{1}} \beta_{1}\left(x_{1}\right),
$$

where $\beta_{1}\left(x_{1}\right)$ is a smooth, non-negative function, yields

$$
\dot{V}_{1} \leq-n x_{1}^{\frac{2 \rho}{r_{1}}}+x_{1}^{\frac{2 \rho-\tau_{1}-r_{1}}{r_{1}}}\left[x_{2}^{p_{1}}-x_{2}^{* p_{1}}\right] .
$$

Inductive Step. Suppose at step $k-1$, there is a $C^{1}$ Lyapunov function $V_{k-1}$ : $\mathbb{R}^{\mathrm{k}-1} \rightarrow \mathbb{R}$, which is positive definite and proper, and a set of $C^{0}$ virtual controllers $x_{1}^{*}, x_{2}^{* p_{1}}, \cdots, x_{k}^{* p_{k-1}}$, defined by

$$
\begin{array}{llc}
x_{1}^{*} & =0 & \xi_{1}=x_{1}^{\frac{\sigma}{r_{1}}}-x_{1}^{*^{\frac{\sigma}{r_{1}}}}, \\
x_{2}^{* p_{1}}=-\xi_{1}^{r_{2} p_{1} / \sigma} \beta_{1}\left(x_{1}\right) & \xi_{2}=x_{2}^{\frac{\sigma}{r_{2}}}-x_{2}^{* \frac{\sigma}{r_{2}}} \\
& \vdots & \vdots \\
x_{k}^{* p_{k-1}}=-\xi_{k-1}^{r_{k} p_{k-1} / \sigma} \beta_{k-1}\left(x_{1}, \ldots, x_{k-1}\right) & \xi_{k}=x_{k}^{\frac{\sigma}{r_{k}}}-x_{k}^{*^{\frac{\sigma}{r_{k}}}},
\end{array}
$$

\footnotetext{
${ }^{1}$ When a $C^{0}$ stabilizer is attained, global strong stability may be the only achievable result. See [15] and the references therein for details of these conditions and global strong stability in the sense of Kurzweil [13].
} 
with smooth functions $\beta_{1}(\cdot)>0, \cdots, \beta_{k-1}(\cdot)>0$ such that

$$
\dot{V}_{k-1} \leq-(n+2-k)\left(\xi_{1}^{\frac{2 \rho}{\sigma}}+\cdots+\xi_{k-1}^{\frac{2 \rho}{\sigma}}\right)+\xi_{k-1}^{\frac{2 \rho-\tau_{k-1}-r_{k-1}}{\sigma}}\left(x_{k}^{p_{k-1}}-x_{k}^{* p_{k-1}}\right) .
$$

It is clear that (10) reduces to the inequality (8) when $k=2$ under the definitions of (9). We claim (10) also holds at step $k$. To prove this, we set

$$
W_{k}=\int_{x_{k}^{*}}^{x_{k}}\left(s^{\frac{\sigma}{r_{k}}}-x_{k}^{* \frac{\sigma}{r_{k}}}\right)^{\frac{2 \rho-\tau_{k}-r_{k}}{\sigma}} d s
$$

and consider the Lyapunov function $V_{k}: \mathbb{R}^{\mathrm{k}} \rightarrow \mathbb{R}$, as

$$
V_{k}\left(x_{1}, \cdots, x_{k}\right)=V_{k-1}\left(x_{1}, \cdots, x_{k-1}\right)+W_{k}\left(x_{1}, \cdots, x_{k}\right),
$$

which works as a $C^{1}$ Lyapunov function based on the following proposition, whose proof is omitted as it is very similar to the proof of Proposition B.1 in [15].

Proposition 2.2. The function $W_{k}\left(x_{1}, \ldots, x_{k}\right)$ defined by (11) is $C^{1}$.

By Proposition 2.2, the derivative of the Lyapunov function $V_{k}$ along (1) is

$$
\begin{aligned}
\dot{V}_{k}= & \dot{V}_{k-1}+\sum_{l=1}^{k-1} \frac{\partial W_{k}}{\partial x_{l}} \dot{x}_{l}+\xi_{k}^{\frac{2 \rho-\tau_{k}-r_{k}}{\sigma}} \dot{x}_{k} \\
\leq & -(n+2-k)\left(\xi_{1}^{\frac{2 \rho}{\sigma}}+\cdots+\xi_{k-1}^{\frac{2 \rho}{\sigma}}\right)+\xi_{k-1}^{\frac{2 \rho-\tau_{k-1}-r_{k-1}}{\sigma}}\left(x_{k}^{p_{k-1}}-x_{k}^{* p_{k-1}}\right) \\
& +\sum_{l=1}^{k-1} \frac{\partial W_{k}}{\partial x_{l}} \dot{x}_{l}+\xi_{k}^{\frac{2 \rho-\tau_{k}-r_{k}}{\sigma}}\left(x_{k+1}^{* p_{k}}+\phi_{k}(\cdot)\right)+\xi_{k}^{\frac{2 \rho-\tau_{k}-r_{k}}{\sigma}}\left(x_{k+1}^{p_{k}}-x_{k+1}^{* p_{k}}\right)
\end{aligned}
$$

for a virtual controller $x_{k+1}^{* p_{k}}$ to be determined later. In order to proceed further, an estimate (in this case, a bounding estimate) for each term in the right hand side of (13) is needed. The following propositions supply these estimates, with their respective proofs located in the Appendix.

Proposition 2.3. There exists a $C^{\infty}$ function $\hat{c}_{k}\left(x_{1}, \ldots, x_{k}\right)>0$ such that

$$
\xi_{k-1}^{\frac{2 \rho-\tau_{k-1}-r_{k-1}}{\sigma}}\left(x_{k}^{p_{k-1}}-x_{k}^{* p_{k-1}}\right) \leq \frac{1}{3} \xi_{k-1}^{\frac{2 \rho}{\sigma}}+\hat{c}_{k}\left(x_{1}, \ldots, x_{k}\right) \xi_{k}^{\frac{2 \rho}{\sigma}} .
$$

Proposition 2.4. There exists a $C^{\infty}$ function $\tilde{b}_{k}\left(x_{1}, \ldots, x_{k}\right)>0$ such that

$$
\xi_{k}^{\frac{2 \rho-\tau_{k}-r_{k}}{\sigma}} \phi_{k}(\cdot) \leq \frac{1}{2}\left(\xi_{1}^{\frac{2 \rho}{\sigma}}+\xi_{2}^{\frac{2 \rho}{\sigma}}+\cdots+\xi_{k-2}^{\frac{2 \rho}{\sigma}}\right)+\frac{1}{3} \xi_{k-1}^{\frac{2 \rho}{\sigma}}+\tilde{b}_{k}\left(x_{1}, \ldots, x_{k}\right) \xi_{k}^{\frac{2 \rho}{\sigma}} .
$$

The third term in (13), namely $\sum_{l=1}^{k-1} \frac{\partial W_{k}}{\partial x_{l}} \dot{x}_{l}$, can now be estimated by the following proposition.

Proposition 2.5. There is a $C^{\infty}$ function $\hat{b}_{k}\left(x_{1}, \ldots, x_{k}\right)>0$ such that

$$
\left|\sum_{l=1}^{k-1} \frac{\partial W_{k}}{\partial x_{l}} \dot{x}_{l}\right| \leq \frac{1}{2}\left(\xi_{1}^{\frac{2 \rho}{\sigma}}+\xi_{2}^{\frac{2 \rho}{\sigma}}+\cdots+\xi_{k-2}^{\frac{2 \rho}{\sigma}}\right)+\frac{1}{3} \xi_{k-1}^{\frac{2 \rho}{\sigma}}+\hat{b}_{k}\left(x_{1}, \ldots, x_{k}\right) \xi_{k}^{\frac{2 \rho}{\sigma}} .
$$


Substituting the results of the previous propositions into (13), we arrive at

$$
\begin{aligned}
\dot{V}_{k} \leq & -(n-k+1)\left(\xi_{1}^{\frac{2 \rho}{\sigma}}+\cdots+\xi_{k-1}^{\frac{2 \rho}{\sigma}}\right) \\
& +\xi_{k}^{\frac{2 \rho-\tau_{k}-r_{k}}{\sigma}} x_{k+1}^{* p_{k}}+\left(\hat{c}_{k}\left(x_{1}, \ldots, x_{k}\right)+\tilde{b}_{k}\left(x_{1}, \ldots, x_{k}\right)+\hat{b}_{k}\left(x_{1}, \ldots, x_{k}\right)\right) \xi_{k}^{\frac{2 \rho}{\sigma}} \\
& +\xi_{k}^{\frac{2 \rho-\tau_{k}-r_{k}}{\sigma}}\left(x_{k+1}^{p_{k}}-x_{k+1}^{* p_{k}}\right) .
\end{aligned}
$$

Observe that a virtual controller of the form

$$
\begin{aligned}
x_{k+1}^{* p_{k}} & =-\xi_{k}^{\frac{r_{k+1} p_{k}}{\sigma}} \beta_{k}\left(x_{1}, \ldots, x_{k}\right) \\
& =-\xi_{k}^{\frac{r_{k+1} p_{k}}{\sigma}}\left[n-k+1+\hat{c}_{k}\left(x_{1}, \ldots, x_{k}\right)+\tilde{b}_{k}\left(x_{1}, \ldots, x_{k}\right)+\hat{b}_{k}\left(x_{1}, \ldots, x_{k}\right)\right],
\end{aligned}
$$

yields

$$
\dot{V}_{k} \leq-(n-k+1) \sum_{i=1}^{k} \xi_{i}^{\frac{2 \rho}{\sigma}}+\xi_{k}^{\frac{2 \rho-\tau_{k}-r_{k}}{\sigma}}\left(x_{k+1}^{p_{k}}-x_{k+1}^{* p_{k}}\right)
$$

This completes the inductive proof. The inductive argument shows that (10) holds for $k=n+1$ with a set of virtual controllers (9). Hence, at the last step, choosing

$$
\begin{aligned}
u & =x_{n+1}=x_{n+1}^{*}=-\xi_{n}^{\left(r_{n}+\tau_{n}\right) / \sigma} \beta_{n}\left(x_{1}, \ldots, x_{n}\right) \\
& =-\beta_{n}\left(x_{n}^{\frac{\sigma}{r_{n}}}+\beta_{n-1}\left(x_{n-1}^{\frac{\sigma}{r_{n}-1}}+\cdots+\beta_{2}\left(x_{2}^{\frac{\sigma}{r_{2}}}+\beta_{1} x_{1}^{\frac{\sigma}{r_{1}}}\right) \cdots\right)\right)^{\frac{r_{n}+\tau_{n}}{\sigma}}
\end{aligned}
$$

yields

$$
\dot{V}_{n} \leq-\left(\xi_{1}^{\frac{2 \rho}{\sigma}}+\cdots+\xi_{n-1}^{\frac{2 \rho}{\sigma}}+\xi_{n}^{\frac{2 \rho}{\sigma}}\right)
$$

where $\dot{V}_{n}<0, \forall x \neq 0$ under $(9)$, and $V_{n}\left(x_{1}, \cdots, x_{n}\right)$ is a positive definite and proper Lyapunov function of the form (12). Thus, (1)-(15) is globally asymptotically stable.

REMARK 2.2. In the case when $\tau_{i}$ is any real number, we are still able to design a feedback controller globally stabilizing the system (1) with necessary modification to preserve the sign of function $[\cdot]^{r_{i} p_{i-1} / \sigma}$, where $\sigma$ is defined as before, though may not be in $\mathbb{R}_{\text {odd }}^{+}$. Specifically, for any real number $r_{i} p_{i-1} / \sigma>0$, we define

$$
[\cdot]^{r_{i} p_{i-1} / \sigma}=\operatorname{sign}(\cdot)|\cdot|^{r_{i} p_{i-1} / \sigma}
$$

Note that this function is differentiable, and for a constant $\gamma \geq 1$,

$$
\frac{\partial}{\partial g} \operatorname{sign}(g)|g|^{\gamma}=\gamma|g|^{\gamma-1}
$$

Using this function, we are able to design the controller without requiring $r_{i} p_{i-1} / \sigma$ to be odd. In this case, the controller can be constructed as

$$
u=-\operatorname{sign}\left(\xi_{n}\right)\left|\xi_{n}\right|^{\left(r_{n}+\tau_{n}\right) / \sigma} \beta_{n}\left(x_{1}, \ldots, x_{n}\right)
$$


with

$$
\begin{array}{ll}
x_{k}^{* p_{k-1}} & =-\operatorname{sign}\left(\xi_{k-1}\right)\left|\xi_{k-1}\right|^{\frac{r_{k} p_{k-1}}{\sigma}} \beta_{k-1}\left(x_{1}, \ldots, x_{k-1}\right), \text { and } \\
\xi_{k} & =\operatorname{sign}\left(x_{k}\right)\left|x_{k}\right|^{\frac{\sigma}{r_{k}}}-\operatorname{sign}\left(x_{k}\right)\left|x_{k}\right|^{*^{\frac{\sigma}{r_{k}}}}, \quad k=2, \cdots, n
\end{array}
$$

where $\xi_{1}=\operatorname{sign}\left(x_{1}\right)\left|x_{1}\right|^{\frac{\sigma}{r_{1}}}$.

REMARK 2.3. Though a homogeneous control law is not the ultimate goal of our approach, the idea of homogeneity is an enabling factor in our ability to guarantee a $C^{1}$ stabilizer in most instances. During the past two decades, the analysis of nonlinear dynamic systems has been studied from the viewpoint of homogeneity and homogeneous systems [8, 1]. Utilizing these notions has allowed the undertaking of the concepts of controllability and controller design for nonlinear systems to be realizable, with the interested reader referred to the works [7, 12, 8, 6, 1] for more detail.

3. Unification of Results in Existence. This section points out the versatility of the previous section's methodology by comprehensively encompassing some notable feedback stabilization schemes whose controllers, using only static feedback, yield starkly different results, namely, smooth [14], non-smooth [15], and finite-time [10] stabilization. Quite noteworthy is the fact that even though these results are special cases of the more generalized version, Theorem 2.1 does not have each one's limitations (most notable is the ability to offer at least $C^{1}$ stabilization where only non-smooth approaches to static state feedback previously existed, as detailed in Section 4).

3.1. Scenario 1: Non-Smooth Stabilization. In this subsection we stabilize system (1) with the restrictions that $p_{i}$ are odd integers and $p_{i} \geq 1$ but now each $\tau_{i}$ is restricted to be equal to zero. In this way, Assumption 2.1 can be seen to transform to the following one.

Assumption 3.1. For $i=1, \cdots, n$, there exists a smooth function $S_{i}(\cdot) \geq 0$ such that

$$
\begin{aligned}
& \left|\phi_{i}(t, x, u)\right| \\
\leq & S_{i}\left(x_{1}, \ldots, x_{i}\right)\left(\left|x_{1}\right|^{\frac{1}{p_{1} \cdots p_{i-1}}}+\left|x_{2}\right|^{\frac{1}{p_{2} \cdots p_{i-1}}}+\cdots+\left|x_{i-1}\right|^{\frac{1}{p_{i-1}}}+\left|x_{i}\right|\right) .
\end{aligned}
$$

Note that for any odd positive integer $p$ and $x \in \mathbb{R}$,

$$
|x| \leq\left(1+x^{2}\right)|x|^{1 / p}
$$

so it can be seen that if the the following is true:

$$
\left|\phi_{i}(t, x, u)\right| \leq \tilde{S}_{i}\left(x_{1}, \ldots, x_{i}\right)\left(\left|x_{1}\right|+\left|x_{2}\right|+\cdots+\left|x_{i}\right|\right)
$$

then

$$
\begin{aligned}
& \left|\phi_{i}(t, x, u)\right| \\
\leq & S_{i}\left(x_{1}, \ldots, x_{i}\right)\left(\left|x_{1}\right|^{\frac{1}{p_{1} \cdots p_{i-1}}}+\left|x_{2}\right|^{\frac{1}{p^{2 \cdots p_{i-1}}}}+\cdots+\left|x_{i-1}\right|^{\frac{1}{p_{i-1}}}+\left|x_{i}\right|\right)
\end{aligned}
$$


also holds, which is an important relation due to the fact that the inequality (19) is utilized in [15] as a consequence of the Taylor expansion of each $C^{1}$ nonlinear perturbation.

Corollary 3.1. When $p_{i} \geq 1$ are odd integers and $\tau_{i}=0, i=1, \ldots, n$, under Assumption 3.1, there exists a feedback controller such that the nonlinear system (1) is guaranteed globally strong stable.

The controller in this case is of the form (15), with $\rho=\sigma=1$ and smooth functions $\beta_{i}=\beta_{i}\left(x_{1}, \ldots, x_{i}\right)$,

$$
u=-\beta_{n}\left(x_{n}^{p_{1} \cdots p_{n-1}}+\beta_{n-1}\left(x_{n-1}^{p_{1} \cdots p_{n-2}}+\cdots+\beta_{2}\left(x_{2}^{p_{1}}+\beta_{1} x_{1}\right) \cdots\right)\right)^{\frac{1}{p_{1} \cdots p_{n-1}}} .
$$

The is obviously a special case of the previous scenario, while also being the problem statement of the work [15] (with $d_{i}(t)=1$ ). As stated in the introduction, this work yields a non-smooth control law, which is what is seen in (21). Interestingly, the approach taken in Theorem 2.1 not only covers this case, but is also not limited to non-smooth stabilization, which will be discussed in more detail in Section 4 .

REMARK 3.1. If we use Scenario 1 with each $p_{i} \in \mathbb{R}_{\text {odd }}^{+}$, then the problem statement of [4], which also yields a non-smooth solution, is additionally covered as another special case of our more general Theorem 2.1, thereby yielding a smoother stabilizer solution for the applicable systems.

3.2. Scenario 2: Smooth Stabilization. In this subsection we stabilize system (1) with the structural requirements that $p_{1} \geq p_{2} \geq \cdots \geq p_{n} \geq 1$ and $\tau_{i}$ are restricted to be equivalent to $p_{i}-1$, which is always either an even value or zero. In this very specialized case, $r_{i}=1$ and Assumption 2.1 can be seen to reduce to the following one.

Assumption 3.2. For $i=1, \cdots, n$,

$$
\left|\phi_{i}(t, x, u)\right| \leq A_{i}\left(x_{1}, \ldots, x_{i}\right)\left(\left|x_{1}\right|^{p_{i}}+\left|x_{2}\right|^{p_{i}}+\cdots+\left|x_{i}\right|^{p_{i}}\right),
$$

for a smooth function $A_{i}(\cdot) \geq 0$.

Corollary 3.2. If $p_{1} \geq p_{2} \geq \cdots \geq p_{n} \geq 1$ and $\tau_{i}=p_{i}-1, i-1, \ldots, n$, then Assumption 3.2 guarantees a state feedback controller such that the nonlinear system (1) is globally asymptotically stable.

A smooth stabilizer will always be seen of the form (15), with $\rho=p_{1}, \sigma=1$, and smooth functions $\beta_{i}\left(x_{1}, \ldots, x_{i}\right)>0$,

$$
\begin{aligned}
u= & -\beta_{n}\left(x_{1}, \ldots, x_{n}\right)\left(x_{n}+\beta_{n-1}\left(x_{1}, \ldots, x_{n-1}\right)\right. \\
& \left.\cdot\left(x_{n-1}+\cdots+\beta_{2}\left(x_{1}, x_{2}\right)\left(x_{2}+\beta_{1}\left(x_{1}\right) x_{1}\right) \cdots\right)\right) .
\end{aligned}
$$

Not so coincidentally, the restriction on the power integrators and Assumption 3.2 are precisely the conditions of the work [14] for $C^{\infty}$ stabilization. 
3.3. Scenario 3: Finite-Time Stabilization. In this subsection we stabilize system (1) with the condition that this stabilization to the origin is accomplished in finite-time. For simplicity we assume that $p_{i}$ are all equal to one, while $\tau_{i}=\tau:=$ $-\frac{2}{2 n+1}$. Therefore, Assumption 2.1 can be seen to transform to the following one.

Assumption 3.3. For $i=1, \cdots, n$, there exists a smooth function $T_{i}(\cdot) \geq 0$ such that

$$
\left|\phi_{i}(t, x, u)\right| \leq T_{i}\left(x_{1}, \ldots, x_{i}\right)\left(\left|x_{1}\right|^{\frac{r_{i}+\tau}{r_{1}}}+\left|x_{2}\right|^{\frac{r_{i}+\tau}{r_{2}}}+\cdots+\left|x_{i}\right|^{\frac{r_{i}+\tau}{r_{i}}}\right),
$$

where the $r_{i}$ are defined in (6).

Note it can be seen that if

$$
\left|\phi_{i}(t, x, u)\right| \leq \tilde{T}_{i}\left(x_{1}, \ldots, x_{i}\right)\left(\left|x_{1}\right|+\left|x_{2}\right|+\cdots+\left|x_{i}\right|\right)
$$

then

$$
\left|\phi_{i}(t, x, u)\right| \leq T_{i}\left(x_{1}, \ldots, x_{i}\right)\left(\left|x_{1}\right|^{\frac{r_{i}+\tau}{r_{1}}}+\left|x_{2}\right|^{\frac{r_{i}+\tau}{r_{2}}}+\cdots+\left|x_{i}\right|^{\frac{r_{i}+\tau}{r_{i}}}\right)
$$

is as well true, which is another important fact since the inequality (25) is used in [10] (replacing their $q_{i}$ with our $r_{i}$ ), though (25) is again a consequence of the Taylor expansion of each $C^{1}$ nonlinear perturbation. (25)-(26) can be easily seen since $\frac{r_{i}+\tau}{r_{j}}<1, j=1, \ldots, i$. Note that [9] stabilized similar systems also in finitetime.

Corollary 3.3. By Assumption 3.3, with $p_{i}=1$ and $\tau_{i}=-\frac{2}{2 n+1}, i=1, \ldots, n$, there exists a feedback controller such that the nonlinear system (1) is guaranteed stable in finite-time.

The controller in this case is of the form (15), with $\rho=2 n /(2 n+1), \sigma=1$ and smooth functions $\beta_{i}=\beta_{i}\left(x_{1}, \ldots, x_{i}\right)$,

$$
u=-\beta_{n}\left(x_{n}^{\frac{1}{r_{n}}}+\beta_{n-1}\left(x_{n-1}^{\frac{1}{r_{n-1}}}+\cdots+\beta_{2}\left(x_{2}^{\frac{1}{r_{2}}}+\beta_{1} x_{1}\right) \cdots\right)\right)^{\frac{1}{2 n+1}} .
$$

Stability is easy to see under this formulation as it is comparable to Scenario 2 . However, stability in finite-time can only be seen by noting the following relations (as in [10])

$$
\begin{aligned}
V_{n}\left(x_{1}, \ldots, x_{n}\right) & \leq 2 \sum_{i=1}^{n} \xi_{i}^{2}, \quad \dot{V}_{n}\left(x_{1}, \ldots, x_{n}\right) \leq-\sum_{i=1}^{n} \xi_{i}^{\frac{4 n}{2 n+1}}, \\
V_{n}^{\frac{2 n}{2 n+1}}\left(x_{1}, \ldots, x_{n}\right) & \leq 2 \sum_{i=1}^{n} \xi_{i}^{\frac{4 n}{2 n+1}} .
\end{aligned}
$$

Putting (28)-(29) together yields

$$
\dot{V}_{n}+\frac{1}{4} V_{n}^{\frac{2 n}{2 n+1}} \leq-\frac{1}{2} \sum_{i=1}^{n} \xi_{i}^{\frac{4 n}{2 n+1}} \leq 0
$$

thus giving the desired result. 


\section{Beyond Non-smooth Stabilization and Structural Constraints for}

Smoothness. In this section we show that the methodology of Theorem 2.1 does not follow the restrictions of the subsequent cases, where smoothness of any form is only seen under strict structural constraints.

Remark 4.1. Note that by the definition of $\sigma$ we know $\frac{\sigma}{r_{k}} \geq 1$. Hence, it can be concluded that the controller $u$, as defined in (15), can be guaranteed to be at least $C^{1}$ when $\frac{r_{n}+\tau_{n}}{\sigma} \geq 1$. Furthermore, when $\frac{\sigma}{r_{k}}, k=1, \cdots, n$, are integers and $\frac{r_{n}+\tau_{n}}{\sigma}=1$, a smooth (i.e. $C^{\infty}$ ) controller is constructed by this design method. Essentially, to achieve at least continuously differentiable stabilization, the existence of such $\sigma$ satisfying $\frac{r_{n}+\tau_{n}}{\sigma} \geq 1$, is guaranteed by the condition

$$
r_{n}+\tau_{n} \geq r_{k}, \quad k=1, \cdots, n .
$$

EXAMPLE 4.1. To see how this condition can be verified, we consider the following planar system

$$
\dot{x}_{1}=x_{2}^{3}+x_{1}^{q}, \quad \dot{x}_{2}=u .
$$

In order to have a $C^{1}$ controller, condition (30) reduces to

$$
r_{2}+\tau_{2}=\left(1+\tau_{1}\right) / 3+\tau_{2} \geq \max \left\{1,\left(1+\tau_{1}\right) / 3\right\}, \quad q \geq 1+\tau_{1} .
$$

A sufficient condition for (32) can be identified as $q \geq 3 / 2$ where we can choose $\tau_{1}=\tau_{2}=1 / 2$.

When $q=1$, system (31) reduces to

$$
\dot{x}_{1}=x_{2}^{3}+x_{1}, \quad \dot{x}_{2}=u,
$$

which cannot be stabilized by any smooth control law (due to the linearized uncontrollable mode with a right-half plane eigenvalue, as detailed in $[11,12,15])$, and so by our methodology we set $\tau_{1}=\tau_{2}=0$ to satisfy Assumption 2.1, thereby necessitating a continuous control law of the form $u=-\beta_{2}\left(x_{2}^{3}+\beta_{1} x_{1}\right)^{1 / 3}$, with positive constants $\beta_{1}$ and $\beta_{2}$. However, in stabilizing the similar system mentioned in the introduction,

$$
\dot{x}_{1}=x_{2}^{3}+x_{1}^{2}, \quad \dot{x}_{2}=u,
$$

since $q=2$, there is an at least $C^{1}$ state feedback controller. Specifically, noting that $x_{1}^{2} \leq\left(1+x_{1}^{2}\right)\left|x_{1}\right|^{5 / 3}=: b_{1}\left(x_{1}\right)\left|x_{1}\right|^{5 / 3}$, a state feedback controller can be designed of the form (by choosing $\tau_{1}=2 / 3, \tau_{2}=4 / 9, r_{1}=1, r_{2}=5 / 9$, and $\sigma=1$ )

$$
u=-\left(20+4 x_{1}^{2}\right)\left(x_{2}^{9 / 5}+\left(3+x_{1}^{2}\right) x_{1}\right),
$$

which globally stabilizes (34) and is a continuously differentiable, or $C^{1}$, stabilizer. Figure 1 illustrates the computer simulation of this stabilization. This ability to create 


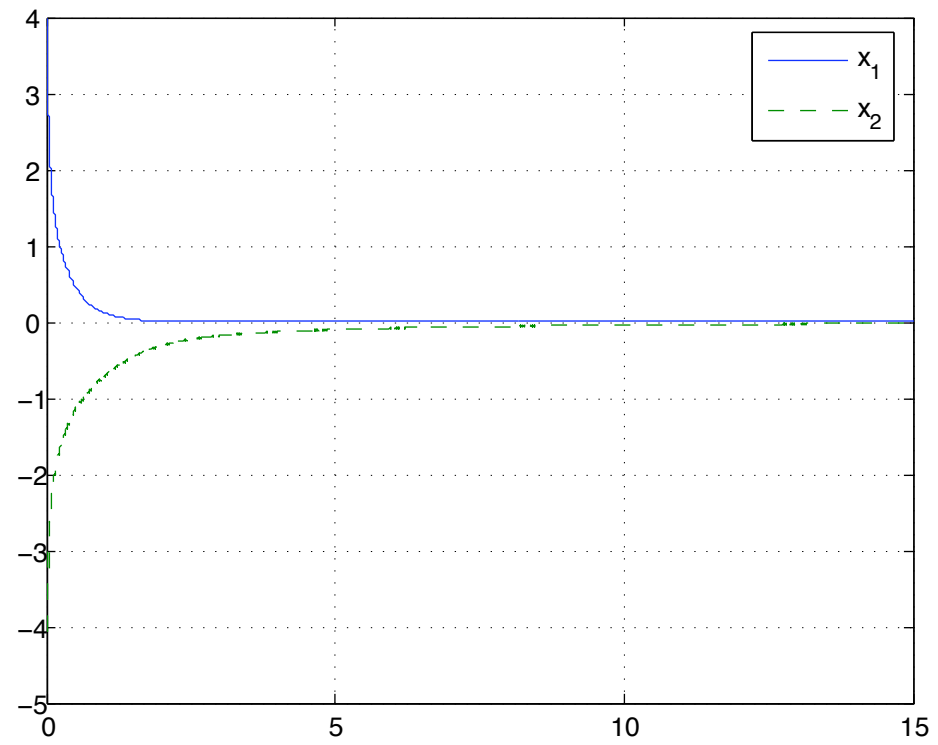

FIG. 1. State trajectories of the closed-loop system (34)-(35) in Example 4.1 with $\left(x_{1}, x_{2}\right)(0)=$ $(4,-3)$.

a $C^{1}$ controller is in sharp contrast to the prior state feedback stabilization results of $[4,15]$, which could only guarantee a non-smooth controller in all cases (33), (34).

Note that since Scenario 1 is a special case of Theorem 2.1, we can use the methodology of Theorem 2.1 as to not limit ourselves to a non-smooth solution when we are not restricted to one by the previously mentioned structural constraints. This point is emphasized by the following example:

EXAMPLE 4.2. Consider the uncertain nonlinear power integrator system

$$
\begin{aligned}
& \dot{x}_{1}=x_{2}+d_{1}(t) e^{x_{1}} x_{1}^{3} \\
& \dot{x}_{2}=x_{3}^{3}+d_{2}(t) x_{1} x_{2}^{4 / 3} \\
& \dot{x}_{3}=u, \quad\left|d_{1}(t)\right| \leq 1, \quad\left|d_{2}(t)\right| \leq 4.5,
\end{aligned}
$$

which is globally strong stabilizable by $C^{0}$ state feedback in [15] and would be reaffirmed by the same type of controller if Scenario 1 (and hence Assumption 3.1 and Corollary 3.2) is used for stabilization. However, there are no structural constraints limiting the stabilizer to be only non-smooth (since the linearization has no uncontrollable modes with positive eigenvalues [2]), thus smooth stabilization may be possible. In investigating this avenue by utilizing the more flexible Theorem 2.1, it can be seen that a controller of the form (by choosing $\tau_{1}=\tau_{2}=\tau_{3}=2$, with $r_{1}=1, r_{2}=3, r_{3}=5 / 3$, and $\left.\sigma=11 / 3\right)$

$$
u=-\beta_{3}\left(x_{1}, x_{2}, x_{3}\right)\left(x_{3}^{11 / 5}+\beta_{2}\left(x_{1}, x_{2}\right)\left(x_{2}^{11 / 9}+\beta_{1}\left(x_{1}\right) x_{1}^{11 / 3}\right)\right)
$$


stabilizes (36) and is $C^{1}$ (with appropriately chosen smooth, strictly positive $\beta_{1}, \beta_{2}$, $\left.\beta_{3}\right)$.

REMARK 4.2. Since a smooth (i.e. $C^{\infty}$ ) stabilizer can be guaranteed whenever the condition $r_{i} \leq r_{n}+\tau_{n}, i=1, \ldots, n$, is met (with all integer values), Scenario 2 implicitly meets this criteria and is thusly a smooth stabilization scenario. See the following example when such a condition is met that cannot be handled by Scenario 2 (and hence [14]), but is still stabilizable by a smooth controller under Theorem 2.1.

EXAMPLE 4.3. Given the nonlinear system

$$
\begin{aligned}
& \dot{x}_{1}=x_{2}^{3} \\
& \dot{x}_{2}=x_{3}^{1 / 3}+d(t) x_{1} x_{2} \\
& \dot{x}_{3}=u, \quad|d(t)| \leq 2,
\end{aligned}
$$

which was previously only stabilizable by a non-smooth controller [4]. But by Theorem 2.1 and Assumption 2.1, system (37) can be globally stabilized by selecting $\tau_{1}=$ $2, \tau_{2}=2 / 3, \tau_{3}=0$ and $r_{1}=r_{2}=1, r_{3}=5$, with $\sigma=5$. Assumption 2.1 still holds since

$$
\left|x_{1} x_{2}\right| \leq \frac{1}{2} x_{1}^{2}+\frac{1}{2} x_{2}^{2} \leq b_{2}\left(x_{1}, x_{2}\right)\left(\left|x_{1}\right|^{5 / 3}+\left|x_{2}\right|^{5 / 3}\right),
$$

for a smooth, non-negative function $b_{2}\left(x_{1}, x_{2}\right)$. Under this formulation our feedback control law is of the form (15) (with smooth $\beta_{1}>0, \beta_{2}>0, \beta_{3}>0$ )

$$
u=-\beta_{3}\left(x_{1}, x_{2}, x_{3}\right)\left(x_{3}+\beta_{2}\left(x_{1}, x_{2}\right)\left(x_{2}^{5}+\beta_{1}\left(x_{1}\right) x_{1}^{5}\right)\right),
$$

which is apparently a $C^{\infty}$ function of the feedback states.

5. Conclusion. To summarize, this paper has accomplished the following:

- The unification of the existing global feedback stabilization literature for nonlinear power integrator systems constituting smooth [14], non-smooth [15], and finite-time [10] solutions;

- The design methodology proposed in Theorem 2.1 of Section 2 was shown to not have the limitations of the previous schemes, with the ability to offer at least $C^{1}$ stabilization (when applicable) where non-smooth stabilization was the previous restriction;

The above properties indeed give credence to the idea of the methodology of Theorem 2.1 as being a universal technique for the robust feedback stabilization of uncertain nonlinear systems.

Appendix. A. Useful Inequalities The next two lemmas, given without proof, were also used in [15] for the implicit tool of adding a power integrator. 
Lemma A.1. For $x \in \mathbb{R}, \mathrm{y} \in \mathbb{R}, p \geq 1$ is a constant, the following inequalities hold:

$$
\begin{aligned}
& |x+y|^{p} \leq 2^{p-1}\left|x^{p}+y^{p}\right|, \\
& (|x|+|y|)^{\frac{1}{p}} \leq|x|^{\frac{1}{p}}+|y|^{\frac{1}{p}} \leq 2^{\frac{p-1}{p}}(|x|+|y|)^{\frac{1}{p}} .
\end{aligned}
$$

If $p \geq 1$ and $p \in \mathbb{R}_{\text {odd }}^{+}$, then

$$
|x-y|^{p} \leq 2^{p-1}\left|x^{p}-y^{p}\right| \quad \text { and } \quad\left|x^{\frac{1}{p}}-y^{\frac{1}{p}}\right| \leq 2^{\frac{p-1}{p}}|x-y|^{\frac{1}{p}} .
$$

Lemma A.2. Let $c, d$ be positive constants. Then, for any real-valued function $\gamma(x, y)>0$, the following inequality holds:

$$
|x|^{c}|y|^{d} \leq \frac{c}{c+d} \gamma(x, y)|x|^{c+d}+\frac{d}{c+d} \gamma^{-\frac{c}{d}(c+d)}(x, y)|y|^{c+d} .
$$

B. Proof of Propositions This part of the appendix contains the technical details of the proofs. Herein we use a generic constant $c$ which exemplifies any finite positive constant value and $c_{i}\left(\bar{x}_{i}\right)=c_{i}\left(x_{1}, \ldots, x_{i}\right)$ represents a smooth, non-negative function, either of which may be implicitly changed in various places. An important notion that is utilized in the following proofs, and should be pointed out, is that there exists a $\delta>0$ such that

$$
x^{2} x^{\delta} \leq x^{2} \alpha(x)
$$

for a smooth, strictly positive function $\alpha(\cdot)$.

Proof of Proposition 2.3: First, whenever $\frac{r_{l} p_{l-1}}{\sigma} \leq 1$, it follows from Lemma A.1 that

$$
\begin{aligned}
\left(x_{l}^{p_{l-1}}-x_{l}^{* p_{l-1}}\right) & \left.\leq \mid\left(x_{l}^{\frac{\sigma}{r_{l}}}\right)^{\frac{r_{l} p_{l-1}}{\sigma}}-\left(x_{l}^{*}\right)^{\frac{\sigma}{r_{l}}}\right)^{\frac{r_{l} p_{l-1}}{\sigma}} \mid \\
& \leq 2^{1-\frac{r_{l} p_{l-1}}{\sigma}}\left|x_{l}^{\frac{\sigma}{r_{l}}}-x_{l}^{* \frac{\sigma}{r_{l}}}\right|^{\frac{r_{l} p_{l-1}}{\sigma}} \\
& \leq 2^{1-\frac{r_{l} p_{l-1}}{\sigma}}\left|\xi_{l}\right|^{\frac{r_{l} p_{l-1}}{\sigma}}=c_{l}\left(\bar{x}_{l}\right)\left|\xi_{l}\right|^{\frac{r_{l} p_{l-1}}{\sigma}} .
\end{aligned}
$$

By the utilization of Lemma A.2 and noting $r_{l} p_{l-1}=\tau_{l-1}+r_{l-1}$, it can be seen that

$$
\begin{aligned}
\xi_{l-1}^{\frac{2 \rho-\tau_{l-1}-r_{l-1}}{\sigma}}\left(x_{l}^{p_{l-1}}-x_{l}^{* p_{l-1}}\right) & \leq \xi_{l-1}^{\frac{2 \rho-\tau_{l-1}-r_{l-1}}{\sigma}} c_{l}\left(\bar{x}_{l}\right)\left|\xi_{l}\right|^{\frac{r_{l} p_{l-1}}{\sigma}} \\
& \leq \frac{1}{3} \xi_{l-1}^{\frac{2 \rho}{\sigma}}+\hat{c}_{l}\left(x_{1}, \ldots, x_{l}\right) \xi_{l}^{\frac{2 \rho}{\sigma}}
\end{aligned}
$$

for a smooth function $\hat{c}_{l}\left(x_{1}, \ldots, x_{l}\right)>0$. However, if $\frac{r_{l} p_{l-1}}{\sigma} \geq 1$, by the Mean Value 
Theorem,

$$
\begin{aligned}
\left(x_{l}^{p_{l-1}}-x_{l}^{* p_{l-1}}\right) & =\left|\left(x_{l}^{\frac{\sigma}{r_{l}}}\right)^{\frac{r_{l} p_{l-1}}{\sigma}}-\left(x_{l}^{*^{\frac{\sigma}{r_{l}}}}\right)^{\frac{r_{l} p_{l-1}}{\sigma}}\right| \\
& \leq c\left|x_{l}^{\frac{\sigma}{r_{l}}}-x_{l}^{*^{\frac{\sigma}{r_{l}}}}\right|\left(\left(x_{l}^{\frac{\sigma}{r_{l}}}\right)^{\frac{r_{l} p_{l-1}}{\sigma}-1}+\left(x_{l}^{*^{\frac{\sigma}{r_{l}}}}\right)^{\frac{r_{l} p_{l-1}}{\sigma}-1}\right) \\
& \leq c_{l}\left(\bar{x}_{l}\right)\left|\xi_{l}\right|\left(\left|\xi_{l}\right|^{\frac{r_{l} p_{l-1}}{\sigma}-1}+\left|\xi_{l-1}\right|^{\frac{r_{l} p_{l-1}}{\sigma}-1}\right),
\end{aligned}
$$

Finally, by Lemma A.2 and again noting that $r_{l} p_{l-1}=\tau_{l-1}+r_{l-1}$, it is apparent that

$$
\begin{aligned}
\xi_{l-1}^{\frac{2 \rho-\tau_{l-1}-r_{l-1}}{\sigma}}\left(x_{l}^{p_{l-1}}-x_{l}^{* p_{l-1}}\right) & \leq \xi_{l-1}^{\frac{2 \rho-\tau_{l-1}-r_{l-1}}{\sigma}} c_{l}\left(\bar{x}_{l}\right)\left|\xi_{l}\right|\left(\left|\xi_{l}\right|^{\frac{r_{l} p_{l-1}}{\sigma}-1}+\left|\xi_{l-1}\right|^{\frac{r_{l} p_{l-1}}{\sigma}-1}\right) \\
\text { (B.4) } & \leq \frac{1}{3} \xi_{l-1}^{\frac{2 \rho}{\sigma}}+\hat{c}_{l}\left(x_{1}, \ldots, x_{l}\right) \xi_{l}^{\frac{2 \rho}{\sigma}}
\end{aligned}
$$

for a smooth function $\hat{c}_{l}\left(x_{1}, \ldots, x_{l}\right)>0$.

Proof of Proposition 2.4: Using Lemma A.1, Assumption 2.1 can be rewritten as $($ for $l=2, \ldots, k)$

$$
\begin{aligned}
\left|\phi_{l}(t, x, u)\right| & \leq b_{l}\left(x_{1}, \ldots, x_{l}\right)\left(\left|\xi_{1}\right|^{\frac{r_{l+1} p_{l}}{\sigma}}+\left|\xi_{2}-\bar{\beta}_{1} \xi_{1}\right|^{\frac{r_{l+1} p_{l}}{\sigma}}+\cdots+\left|\xi_{l}-\bar{\beta}_{l} \xi_{l}\right|^{\frac{r_{l+1} p_{l}}{\sigma}}\right) \\
\text { (B.5) } & \leq \bar{b}_{l}\left(x_{1}, \ldots, x_{l}\right)\left(\left|\xi_{1}\right|^{\frac{r_{l+1} p_{l}}{\sigma}}+\left|\xi_{2}\right|^{\frac{r_{l+1} p_{l}}{\sigma}}+\cdots+\left|\xi_{l}\right|^{\frac{r_{l+1} p_{l}}{\sigma}}\right)
\end{aligned}
$$

for smooth, positive nonzero functions $\bar{\beta}_{i}\left(x_{1}, \ldots, x_{i}\right):=\beta_{i}^{\sigma / r_{i} p_{i-1}}\left(x_{1}, \ldots, x_{i}\right), i=$ $1, \ldots, l$, and $\bar{b}_{l}\left(x_{1}, \ldots, x_{l}\right)$. By Lemma A.2 and (B.5), (with $\left.\frac{2 \rho-\tau_{l}-r_{l}}{\sigma}+\frac{r_{l+1} p_{l}}{\sigma}=\frac{2 \rho}{\sigma}\right)$

$$
\begin{aligned}
\xi_{l}^{\frac{2 \rho-\tau_{l}-r_{l}}{\sigma}} \phi_{l}(\cdot) & \leq\left|\xi_{l}\right|^{\frac{2 \rho-\tau_{l}-r_{l}}{\sigma}} \bar{b}_{l}\left(x_{1}, \ldots, x_{l}\right) \sum_{i=1}^{l}\left|\xi_{i}\right|^{\frac{r_{l+1} p_{l}}{\sigma}} \\
& \leq \frac{1}{2}\left(\xi_{1}^{\frac{2 \rho}{\sigma}}+\xi_{2}^{\frac{2 \rho}{\sigma}}+\cdots+\xi_{l-2}^{\frac{2 \rho}{\sigma}}\right)+\frac{1}{3} \xi_{l-1}^{\frac{2 \rho}{\sigma}}+\tilde{b}_{l}\left(x_{1}, \ldots, x_{l}\right) \xi_{l}^{\frac{2 \rho}{\sigma}}
\end{aligned}
$$

for a smooth function $\tilde{b}_{l}(\cdot)>0$.

Proof of Proposition 2.5: First, it can be seen that for $l=1, \cdots, k-1$

$$
\begin{aligned}
\left|\frac{\partial W_{k}}{\partial x_{l}} \dot{x}_{l}\right| & \leq c\left|x_{k}-x_{k}^{*}\right|\left|\xi_{k}\right|^{\frac{2 \rho-\tau_{k}-r_{k}}{\sigma}}-1\left|\frac{\partial x_{k}^{* \frac{\sigma}{r_{k}}}}{\partial x_{l}} \dot{x}_{l}\right| \\
& \leq c\left|\xi_{k}\right|^{\frac{2 \rho-\tau_{k}-\sigma}{\sigma}}\left|\frac{\partial x_{k}^{* \frac{\sigma}{r_{k}}}}{\partial x_{l}} \dot{x}_{l}\right|
\end{aligned}
$$

where the last inequality is from (A.3) with $p=\frac{\sigma}{r_{k}} \geq 1$. By definition of $x_{k}^{*}$ and 
(A.2),

$$
\begin{aligned}
\frac{\partial x_{k}^{\frac{\sigma}{r_{k}}}}{\partial x_{l}} & =\frac{\partial\left(\bar{\beta}_{k-1}\left(x_{1}, \ldots, x_{k-1}\right) \xi_{k-1}\right)}{\partial x_{l}} \\
& =c_{k-1}\left(\bar{x}_{k-1}\right) \frac{\partial\left(x_{l}^{\frac{\sigma}{r_{l}}}\right)}{\partial x_{l}}=c_{k-1}\left(\bar{x}_{i}\right) x_{l}^{\frac{\sigma-r_{l}}{r_{l}}}=c_{k-1}\left(\bar{x}_{k-1}\right)\left(\xi_{l}+x_{l}^{*^{\frac{\sigma}{r_{l}}}}\right)^{\frac{\sigma-r_{l}}{\sigma}} \\
& =c_{k-1}\left(\bar{x}_{k-1}\right)\left(\xi_{l}+\bar{\beta}_{l-1} \xi_{l-1}\right)^{\frac{\sigma-r_{l}}{\sigma}} \leq c_{k-1}\left(\bar{x}_{k-1}\right) \sum_{i=l-1}^{l}\left|\xi_{i}\right|^{\frac{\sigma-r_{l}}{\sigma}} .
\end{aligned}
$$

This, together with (B.5) gives

$$
\begin{aligned}
\left|\frac{\partial x_{k}^{*^{\frac{\sigma}{r_{k}}}}}{\partial x_{l}} \dot{x}_{l}\right| & \leq c_{k-1}\left(\bar{x}_{k-1}\right) \sum_{i=l-1}^{l}\left|\xi_{i}\right|^{\frac{\sigma-r_{l}}{\sigma}}\left(\left|x_{l+1}\right|^{p_{l}}+\sum_{j=1}^{l}\left|\xi_{j}\right|^{\frac{r_{l+1} p_{l}}{\sigma}}\right) \\
& \leq c_{k-1}\left(\bar{x}_{k-1}\right) \sum_{i=l-1}^{l}\left|\xi_{i}\right|^{\frac{\sigma-r_{l}}{\sigma}}\left(\left|\xi_{l+1}+x_{l+1}^{*^{\frac{\sigma}{r_{l+1}}}}\right|^{\frac{r_{l+1} p_{l}}{\sigma}}+\sum_{j=1}^{l}\left|\xi_{j}\right|^{\frac{r_{l+1} p_{l}}{\sigma}}\right) \\
& \leq c_{k-1}\left(\bar{x}_{k-1}\right) \sum_{i=l-1}^{l}\left|\xi_{i}\right|^{\frac{\sigma-r_{l}}{\sigma}}\left(\left|\xi_{l+1}\right|^{\frac{r_{l+1} p_{l}}{\sigma}}+\left|\xi_{l}\right|^{\frac{r_{l+1} p_{l}}{\sigma}}+\sum_{j=1}^{l}\left|\xi_{j}\right|^{\frac{r_{l+1} p_{l}}{\sigma}}\right) .
\end{aligned}
$$

By Lemma A.2 and the fact that $r_{l+1} p_{l}=\tau_{l}+r_{l}$, we have

$$
\left|\frac{\partial x_{k}^{*^{\frac{\sigma}{r_{k}}}}}{\partial x_{l}} \dot{x}_{l}\right| \leq c_{k-1}\left(\bar{x}_{k-1}\right) \sum_{i=1}^{l+1}\left|\xi_{i}\right|^{\frac{\sigma-r_{l}+r_{l+1} p_{l}}{\sigma}}=c_{k-1}\left(\bar{x}_{k-1}\right) \sum_{i=1}^{l+1}\left|\xi_{i}\right|^{\frac{\sigma+\tau_{l}}{\sigma}} .
$$

Under the realization that $\tau_{l} \geq \tau_{k}, \forall l=1, \ldots, k-1$, the following can be seen:

$$
\begin{aligned}
\left|\frac{\partial W_{k}}{\partial x_{l}} \dot{x}_{l}\right| & \leq c_{k-1}\left(\bar{x}_{k-1}\right)\left|\xi_{k}\right|^{\frac{2 \rho-\tau_{k}-\sigma}{\sigma}}\left|\frac{\partial x_{k}^{\frac{\sigma}{r_{k}}}}{\partial x_{l}} \dot{x}_{l}\right| \\
& \leq c_{k-1}\left(\bar{x}_{k-1}\right)\left|\xi_{k}\right|^{\frac{2 \rho-\tau_{k}-\sigma}{\sigma}} \sum_{i=1}^{l+1}\left|\xi_{i}\right|^{\frac{\sigma+\tau_{l}}{\sigma}} \\
& \leq \frac{1}{2}\left(\xi_{1}^{\frac{2 \rho}{\sigma}}+\xi_{2}^{\frac{2 \rho}{\sigma}}+\cdots+\xi_{k-2}^{\frac{2 \rho}{\sigma}}\right)+\frac{1}{3} \xi_{k-1}^{\frac{2 \rho}{\sigma}}+\hat{b}_{k}\left(x_{1}, \ldots, x_{k}\right) \xi_{k}^{\frac{2 \rho}{\sigma}}
\end{aligned}
$$

by Lemma A.2 and for a smooth, strictly positive function $\hat{b}_{k}\left(x_{1}, \ldots, x_{k}\right)$.

\section{REFERENCES}

[1] A. Bacciotti and L. Roiser, Liapunov Functions and Stability in Control Theory, volume 267 of Lecture Notes in Control and Information Sciences. Springer, 2001.

[2] R. W. Brockett, Asymptotic stability and feedback stabilization, In: Differential geometric control theory (Houghton, Mich., 1982), volume 27 of Progr. Math., pages 181-191. Birkhäuser Boston, Boston, MA, 1983. 
[3] S. Celikovsky and E. Aranda-Bricaire, Constructive nonsmooth stabilization of triangular systems, Systems Control Lett., 36:1(1999), pp. 21-37.

[4] S. Cheong, J. Back, H. Shim, And J. Seo, Non-smooth feedback stabilizer for strict-feedback nonlinear systems not even linearizable at the origin, In: Proceedings of 2005 American Control Conference, pages 1907-1912, June 2005.

[5] J.-M. Coron and L. Praly, Adding an integrator for the stabilization problem, Systems Control Lett., 17:2(1991), pp. 89-104.

[6] W. P. Dayawansa, Recent advances in the stabilization problem for low dimensional systems, In: Proceedings of 1992 IFAC NOLCOS, pages 1-8, 1992.

[7] W. P. Dayawansa, C. F. Martin, and G. Knowles, Asymptotic stabilization of a class of smooth two-dimensional systems, SIAM J. Control Optim., 28:6(1990), pp. 1321-1349.

[8] H. Hermes, Homogeneous coordinates and continuous asymptotically stabilizing feedback controls, In: Differential equations (Colorado Springs, CO, 1989), volume 127 of Lecture Notes in Pure and Appl. Math., pages 249-260. Dekker, New York, 1991.

[9] Y. Hong, Finite-time stabilization and stabilizability of a class of controllable systems, Systems and Control Letters, 46(2002), pp. 231-236.

[10] X. Huang, W. Lin, And B. YAng, Global finite-time stabilization of a class of uncertain nonlinear systems, Automatica, 41(2005), pp. 881-888.

[11] M. Kawski, Stabilization of nonlinear systems in the plane, Systems Control Lett., 12:2(1989), pp. 169-175.

[12] M. Kawski, Homogeneous stabilizing feedback laws, Control Theory Adv. Tech., 6:4(1990), pp. 497-516.

[13] J. KurzWeIL, On the inversion of lyapunov's second theorem on the stability of motion, AMST, pages 19-77, 1956.

[14] W. Lin And C. QIAN, Adding one power integrator: a tool for global stabilization of high-order lower-triangular systems, Systems Control Lett., 39:5(2000), pp. 339-351.

[15] C. QIAn And W. Lin, A continuous feedback approach to global strong stabilization of nonlinear systems, IEEE Trans. Automat. Control, 46:7(2001), pp. 1061-1079.

[16] C. Rui, M. Reyhangolu, I. Kolmanovsky, S. Cho, and H. N. McClamroch, Non smooth stabilization of an underactuated unstable two degrees of freedom mechnical system, In: IEEE CDC, volume 4, pages 3998-4003, 1997.

[17] J. Tsinias, Global extension of coron-praly theorem on stabilization for triangular systems, ECC, pages 1834-1839, 1997.

[18] M. Tzamtzi and J. Tsinias, Explicit formulas of feedback stabilizers for a class of triangular systems with uncontrollable linearization, Systems Control Lett., 38:2(1999), pp. 115-126. 